\title{
Atmospheric oscillations in solar magnetic flux tubes
}

\section{Excitation by longitudinal tube waves and random pulses}

\author{
Z. E. Musielak ${ }^{1,2}$ and P. Ulmschneider ${ }^{2}$ \\ 1 Department of Physics, University of Texas at Arlington, Arlington, TX 76019, USA \\ 2 Institut für Theoretische Astrophysik der Universität Heidelberg, Tiergartenstr. 15, 69121 Heidelberg, Germany
}

Received 23 July 2002 / Accepted 7 January 2003

\begin{abstract}
The response of exponentially spreading magnetic flux tubes embedded in an isothermal solar atmosphere to the propagation of longitudinal tube waves and random pulses produced in the solar convection zone is studied analytically. General solutions are presented and applied to solar tube models. It is shown that free atmospheric oscillations inside these flux tubes are generated with oscillation periods near $3 \mathrm{~min}$, which are essentially identical to the oscillation periods observed in the interior regions of supergranulation cells. The observed oscillations are therefore consistent with processes in magnetic tubes as well as in nonmagnetic regions. Stochastic perpetual excitation is expected to keep these flux tube oscillations present at all times. These oscillations are inconsistent with the observed 7-min oscillations in the chromospheric network.
\end{abstract}

Key words. Sun: photosphere - Sun: chromosphere - Sun: oscillations - physical data and processes: MHD physical data and processes: waves

\section{Introduction}

The outer solar atmosphere is known to oscillate with a different period than the solar photosphere. While the latter is dominated by $5 \mathrm{~min}$ oscillations, the main oscillations of the solar chromosphere are near 3 min (see Deubner 1991; Rutten \& Uitenbroek 1991; Curdt \& Heinzel 1998). From a theoretical point of view, there are fundamental differences in the origin of these oscillations. The solar 5-min oscillations are caused by the so-called "cavity modes", which are essentially acoustic waves trapped inside the Sun (e.g., Ulrich 1970; Leibacher \& Stein 1971). However, the solar 3-min oscillations are likely to be a response of the solar chromosphere to propagating acoustic waves, which not only heat the chromosphere (e.g., Ulmschneider et al. 2001) but also excite free atmospheric oscillations at the acoustic cutoff frequency (see Fleck \& Schmitz 1991).

Extensive analytical (Fleck \& Schmitz 1993; Kalkofen et al. 1994; Schmitz \& Fleck 1995; Sutmann et al. 1998) and numerical (Sutmann \& Ulmschneider 1995a, b) studies have been performed of the behaviour of these waves in the solar atmosphere. The main obtained results can be summarized as follows. The free atmospheric oscillations are always present, independent of the form of the initial disturbance that caused them, and they decay in time as $t^{-3 / 2}$ if the frequency of the driving waves is not equal to the acoustic cutoff frequency. Obviously, there are also so-called forced atmospheric oscillations, which represent either propagating or evanescent

Send offprint requests to: $\mathrm{P}$. Ulmschneider, e-mail: ulmschneider@ita.uni-heidelberg.de acoustic waves, and do not decay in time if the wave source drives them continuously. Finally, in the special case when the wave frequency is exactly equal to the cutoff frequency, the free and forced oscillations are the same and they do not decay in time.

The above described 3-min atmospheric oscillations are seen in the interior of the supergranulation cells. It is observationally well established that isolated strong vertical magnetic fields (flux tubes) exist outside sunspots, particularly at the boundaries of supergranulation cells (e.g., Solanki 1993; Stenflo 1994), and there they give rise to the chromospheric network emission in the observed chromospheric spectral lines. The tubes are "windows" which channel the wave energy generated in the solar convection zone to the solar atmosphere (e.g., Spruit \& Roberts 1983; Musielak et al. 1989) and form the chromospheric magnetic network of the quiet Sun. Observations have shown that atmospheric oscillations in this network have a period near 7 min (Damé 1983; Lites et al. 1993; Curdt \& Heinzel 1998).

Because magnetic flux tubes permit the propagation of three types of magnetohydrodynamic waves, longitudinal, transverse and torsional waves (Solanki 1993; Narain \& Ulmschneider 1996), it would be interesting to know if these wave modes are able to generate oscillations and whether the mentioned observations could be used to identify the wave modes. Kalkofen (1997) suggested that propagating transverse tube waves cause the flux tubes to oscillate with the cutoff frequency for these waves. This has been further investigated by Hasan \& Kalkofen (1999) who considered the generation of 
transverse tube waves by the buffeting action of granules on the tubes. The main conclusion of their paper is that the wave energy fluxes carried by transverse tube waves are in agreement with the observed chromospheric power spectrum of network oscillations. The authors also suggest a possible role played by longitudinal tube waves in these atmospheric oscillations.

In this and a subsequent paper (Musielak \& Ulmschneider 2003, Paper II) we want to provide a partial answer to the question whether propagating longitudinal (this paper) or transverse (Paper II) waves give rise to the kind of oscillations which have been observed. In order to keep the argument simple, we suppose a very idealized model of an isolated isothermal exponentially spreading flux tube and compute the time-dependent evolution of various wave-like disturbances analytically, assuming adiabatic wave propagation. We think that the disturbances, either waves or pulses, are generated by the external turbulent motions in the solar convection zone; note that the interaction between the solar $p$-modes and magnetic flux tubes (see Bogdan 1992, 1994, and references therein) is not included in our approach.

The problem is approached by casting the derived wave equation for longitudinal tube waves into a Klein-Gordon form, which shows explicitly the cutoff frequency (Sect. 2) and is solved by using a Laplace transformation (Sect. 3). Subsequently the obtained analytical solutions are applied to our simplified magnetic flux tube model and the time evolution of the generated free atmospheric oscillations are derived and discussed (Sect. 4); in these applications, we use the most recently computed wave energy spectra for longitudinal tube waves (Musielak et al. 1995, 2000, 2002). Discussion of the obtained results and our final conclusions are given in Sect. 5.

\section{Klein-Gordon equation and cutoff frequency}

We consider an isolated magnetic flux tube embedded in a magnetic field-free compressible and isothermal medium. The tube is assumed to be thin, untwisted, and oriented vertically, with circular cross-section, and in temperature equilibrium with its surroundings. We choose a Cartesian coordinate system with the $z$-axis along the tube axis and gravity $\boldsymbol{g}=-g \hat{z}$, where $\hat{z}$ is the unit vector along the $z$-axis. Because of the thin flux tube approximation, the magnetic field inside the tube is given by $\boldsymbol{B}_{\mathrm{o}}=B_{\mathrm{o}}(z) \hat{z}$. In addition, $\boldsymbol{B}_{\mathrm{e}}=0$ and we assume horizontal pressure balance $p_{\mathrm{o}}+B_{\mathrm{o}}^{2} / 8 \pi=p_{\mathrm{e}}$, where $p_{\mathrm{o}}$ and $p_{\mathrm{e}}$ are the internal and external gas pressures; note that subscripts "o" and "e" will be used throughout this paper to denote the internal and external tube parameters, respectively. Magnetic flux conservation and the horizontal pressure balance lead to an exponentially spreading tube geometry.

Solar observations show that outside sunspots an isolated tube has magnetic field strengths of around $B_{\mathrm{o}}=1500 \mathrm{G}$ at $\tau_{5000}=1$ (e.g., Solanki 1993), which approximately corresponds to $B_{\mathrm{o}}=0.85 B_{\mathrm{eq}}$, where $B_{\mathrm{eq}}=\sqrt{p_{\mathrm{e}} / 8 \pi}$ is the equipartition field strength. This type of field is usually found for magnetic elements in the chromospheric network at the boundary of supergranulation cells. Because one observes different field strengths of the various tubes on the Sun we adopt tube models with field strengths varying from $B_{\mathrm{o}}=0.75 B_{\text {eq }}$ to $B_{\mathrm{o}}=0.95 B_{\text {eq }}$ at the solar surface. Our exponentially spreading tube models are not ideal for a crowded network situation because they will expand very quickly and run into neighboring flux tubes, from where they are prevented to spread further. So our results for greater heights may be inapplicable for some cases. Another situation for which our model should be valid is for isolated intranetwork fields. For these, Wang et al. (1995) found typical magnetic fluxes of $6 \times 10^{16} \mathrm{Mx}$ which assuming tube radii of $40 \mathrm{~km}$ at the solar surface would have similar field strengths as taken for the network tubes.

To describe the waves or pulses in our tube model, we introduce the velocity perturbation, $\boldsymbol{v}=v_{\mathrm{z}}(z, t) \hat{z}$, the magnetic field perturbation, $\boldsymbol{b}=b_{\mathrm{z}}(z, t) \hat{z}$, the density perturbation, $\rho=\rho(z, t)$, and the pressure perturbation, $p=p(z, t)$. Then, we linearize the basic MHD equations, apply the thin flux tube approximation, and derive the wave equation for the velocity perturbation (e.g., Musielak et al. 1989)

$\frac{\partial^{2} v_{\mathrm{z}}}{\partial t^{2}}-c_{T}^{2} \frac{\partial^{2} v_{\mathrm{z}}}{\partial z^{2}}+\frac{c_{\mathrm{T}}^{2}}{2 H} \frac{\partial v_{\mathrm{z}}}{\partial z}+\frac{c_{\mathrm{T}}^{2}}{H^{2}}\left(\frac{1}{2}-\frac{1}{2 \gamma}+\frac{c_{\mathrm{S}}^{2}}{c_{\mathrm{A}}^{2}} \frac{\gamma-1}{\gamma^{2}}\right) v_{\mathrm{z}}=0$,

where the tube velocity is given by

$c_{\mathrm{T}}=\frac{c_{\mathrm{S}} c_{\mathrm{A}}}{\sqrt{c_{\mathrm{S}}^{2}+c_{\mathrm{A}}^{2}}}$

and $c_{\mathrm{S}}=\gamma p_{\mathrm{o}} / \rho_{\mathrm{o}}$ is the sound speed, $c_{\mathrm{A}}=B_{\mathrm{o}} / \sqrt{4 \pi \rho_{\mathrm{o}}}$ the Alfvén velocity, and $H$ the pressure scale height. Since in our model both $c_{\mathrm{S}}$ and $c_{\mathrm{A}}$ are constant, the tube velocity $c_{\mathrm{T}}$ is also constant and the form of the derived wave equation is the same for every wave variable.

To cast Eq. (1) in the form of a Klein-Gordon equation, we introduce $v_{\mathrm{z}}(z, t)=v(z, t) \sqrt{B_{\mathrm{o}} / \rho_{\mathrm{o}}}$ and obtain

$\left[\frac{\partial^{2}}{\partial t^{2}}-c_{\mathrm{T}}^{2} \frac{\partial^{2}}{\partial z^{2}}+\Omega_{\mathrm{T}}^{2}\right] v(z, t)=0$,

where $\Omega_{\mathrm{T}}$ is the cutoff frequency for longitudinal tube waves (Defouw 1976)

$\Omega_{\mathrm{T}}=\frac{c_{\mathrm{T}}}{H}\left(\frac{9}{16}-\frac{1}{2 \gamma}+\frac{c_{\mathrm{S}}^{2}}{c_{\mathrm{A}}^{2}} \frac{\gamma-1}{\gamma^{2}}\right)^{1 / 2}$,

which is the frequency of the free atmospheric oscillations inside the magnetic flux tube. The fact that the wave equation for longitudinal tube waves can be transformed into a Klein-Gordon equation was first recognized by Rae \& Roberts (1982) in their studies of the propagation of these waves in the solar atmosphere; a similar approach was used independently by Musielak et al. $(1989,1995)$ who investigated the efficiency of generation of longitudinal tube waves in the solar and stellar convection zones.

The characteristic speed $c_{\mathrm{T}}$ and the Alfvén speed $c_{\mathrm{A}}$ for longitudinal tube waves can be expressed in terms of the sound speed $c_{\mathrm{S}}$ and the plasma $\beta=8 \pi p_{\mathrm{o}} / B_{\mathrm{o}}^{2}$ as

$c_{\mathrm{T}}=\frac{c_{\mathrm{S}}}{\sqrt{1+\gamma \beta / 2}}, \quad c_{\mathrm{A}}=c_{\mathrm{S}} \sqrt{\frac{2}{\gamma \beta}}$,

which shows that $c_{\mathrm{T}}$ is always lower than the sound speed. It approaches $c_{\mathrm{S}}$ as $\beta \rightarrow 0$, and decreases with increasing $\beta$. 
Using the above relations, we may express the cutoff frequency $\Omega_{\mathrm{T}}$ in terms of the acoustic cutoff frequency $\Omega_{\mathrm{S}}=$ $c_{\mathrm{S}} / 2 H$ (Lamb 1908) and the plasma $\beta$. This gives

$\Omega_{\mathrm{T}}=\frac{\Omega_{\mathrm{S}}}{\sqrt{1+\gamma \beta / 2}}\left(\frac{9}{4}-\frac{2}{\gamma}+2 \beta \frac{\gamma-1}{\gamma}\right)^{1 / 2}$.

In the limit $\beta \rightarrow 0$, we have $\Omega_{\mathrm{T}} / \Omega_{\mathrm{S}} \rightarrow \sqrt{9 / 4-2 / \gamma} \approx 1.025$, however, for $\beta \gg 0$, one finds $\sqrt{4(\gamma-1)} / \gamma \approx 0.98$. This shows that $\Omega_{\mathrm{T}}$ is always comparable to $\Omega_{\mathrm{S}}$ and that the range of frequencies for the propagation of both waves is very similar (see Sect. 4, for more discussion).

Note also that $\Omega_{\mathrm{T}}$ and $\Omega_{\mathrm{S}}$ are the only cutoff frequencies in the approach presented here. Hence, longitudinal tube waves are propagating if their frequency $\omega>\Omega_{\mathrm{T}}$, otherwise they are evanescent. The cutoff frequency $\Omega_{\mathrm{S}}$ plays the same role for the propagation of acoustic waves in the external medium. Clearly, the cutoff frequency $\Omega_{\mathrm{T}}$ is not as simple as for acoustic waves and it depends on both pressure (density) and magnetic field scale heights as well as on the Brunt-Väisälä frequency modified by the tube geometry (e.g., Musielak et al. 1989; also Hasan \& Kalkofen 1999).

\section{Mathematical solutions}

To solve the derived Klein-Gordon equation we must specify the initial and boundary conditions. Following Sutmann et al. (1998), we take

$$
\begin{array}{lrl}
\lim _{t \rightarrow 0} v(t, z) & =0, & \lim _{t \rightarrow 0, z \neq 0} \frac{\partial v}{\partial t}=0, \\
\lim _{z \rightarrow 0} v(t, z)=v_{0}(t), & \lim _{z \rightarrow \infty} v(t, z)=0,
\end{array}
$$

where $v_{0}(t)$ is an arbitrary excitation velocity to be prescribed at $z=0$ inside the tube. By specifying these initial and boundary conditions, we solve the homogeneous Klein-Gordon Eq. (3) with the inhomogeneous boundary conditions (8). For different ways of solving this problem see Davies (1978), Haberman (1987) and Edwards \& Penny (1989).

Since the derived Klein-Gordon equation and the prescribed initial and boundary conditions are of the same form as those considered by Sutmann et al. (1998) for acoustic waves, their results can be directly used here. Thus, the general solution is

$v(t, z)=v_{\mathrm{o}}\left(t-z / c_{\mathrm{T}}\right) \mathcal{H}\left(t-z / c_{\mathrm{T}}\right)+\int_{0}^{t} v_{\mathrm{o}}(t-\tau) W(\tau, z) \mathrm{d} \tau$,

where $\mathcal{H}\left(t-z / c_{\mathrm{T}}\right)$ is the Heaviside step-function which has the value is 0 for $t<z / c_{\mathrm{T}}$ and 1 for all values of $t>z / c_{\mathrm{T}}$, while $\mathcal{H}\left(t-z / c_{\mathrm{T}}\right)=0.5$ if $t=z / c_{\mathrm{T}}$. In addition, $W(\tau, z)$ is given by

$W(\tau, z)=-\frac{\Omega_{\mathrm{T}}}{c_{\mathrm{T}}} \frac{J_{1}\left(\Omega_{\mathrm{T}} \sqrt{\tau^{2}-\left(z / c_{\mathrm{T}}\right)^{2}}\right) z}{\sqrt{\tau^{2}-\left(z / c_{\mathrm{T}}\right)^{2}}} \mathcal{H}\left(\tau-z / c_{\mathrm{T}}\right)$.

Note that the solution for $v(t, z)$ is obtained by using the Laplace transform method (see Sutmann et al. 1998, for more details).

We now consider four different types of excitation of the free and forced oscillations inside the magnetic flux tube, namely, excitation by monochromatic longitudinal tube waves, a spectrum of longitudinal tube waves, a $\delta$-function pulse and a wavetrain of random pulses. Analytical solutions to all these cases have already been obtained by Sutmann et al. (1998) for acoustic waves propagating in an isothermal atmosphere. An interesting result of the present paper is that the mathematical structure of the basic equations derived here for longitudinal tube waves and those obtained by Sutmann et al. for acoustic waves is the same, which means that the solutions for both types of waves become identical when $c_{\mathrm{S}}$ is replaced by $c_{\mathrm{T}}$ and $\Omega_{\mathrm{S}}$ by $\Omega_{\mathrm{T}}$. In the following, we write only the final solutions without repeating the detailed derivations. These solutions will be then used to study the free atmospheric oscillations of magnetic flux tubes in the solar atmosphere (see Sect. 4).

\subsection{Excitation by monochromatic longitudinal tube waves}

We consider a monochromatic longitudinal tube wave with frequency $\omega$ and amplitude $u_{\mathrm{o}}$, and assume that these waves are continuously generated by the external turbulent motions (see Sect. 4) and that they propagate along the flux tube. The required boundary condition (Eq. (8)) is

$v_{\mathrm{o}}(t)=u_{\mathrm{o}} \mathrm{e}^{-\mathrm{i} \omega t}$,

which allows writing the general solution (see Eq. (27) in Sutmann et al. 1998) as

$$
\begin{aligned}
v(t, z)= & u_{\mathrm{o}} \mathrm{e}^{-\mathrm{i}\left(\omega t-\sqrt{\omega^{2}-\Omega_{\mathrm{T}}^{2}} z / c_{\mathrm{T}}\right)}+u_{\mathrm{o}} \sqrt{\frac{2 \Omega_{\mathrm{T}}}{\pi}} \frac{1}{\omega^{2}-\Omega_{\mathrm{T}}^{2}} \frac{z}{c_{\mathrm{T}}} \frac{1}{t^{3 / 2}} \\
& \times\left[\Omega_{\mathrm{T}} \sin \left(\Omega_{\mathrm{T}} t-\frac{3 \pi}{4}\right)+\mathrm{i} \omega \cos \left(\Omega_{\mathrm{T}} t-\frac{3 \pi}{4}\right)\right] .
\end{aligned}
$$

According to this formula, the response of the atmosphere inside the flux tube to the propagating monochromatic waves is a superposition of two different types of oscillations: forced atmospheric oscillations with the wave frequency $\omega$ and free atmospheric oscillations with the cutoff frequency $\Omega_{\mathrm{T}}$. The forced oscillations with the driving frequency $\omega$ represent propagating longitudinal tube waves if $\omega>\Omega_{\mathrm{T}}$ and evanescent waves if $\omega \leq \Omega_{\mathrm{T}}$; the behavior of these waves in both frequency regimes is well-known (Defouw 1976) and they do not decay in time because they are driven continuously by the wave source. The free oscillations are described by the second term on the RHS of Eq. (12) and it is seen that they decay in time as $t^{-3 / 2}$ at any given height; in addition, their amplitude increases linearly with height.

This phenomenon has been extensively studied in the literature for acoustic waves (e.g., Fleck \& Schmitz 1991, 1993; Kalkofen et al. 1994; Schmitz \& Fleck 1995; Sutmann et al. 1998). It has been expected that similar oscillations driven by longitudinal tube waves and decaying in time as $t^{-3 / 2}$ will also exist inside solar magnetic flux tubes (e.g., Hasan \& Kalkofen 1999, and references therein); the analytical solutions presented in this paper clearly show that this is indeed the case.

It must be noted that the free atmospheric oscillations with the acoustic cutoff frequency have also been referred to as 
resonance oscillations (e.g., Fleck \& Schmitz 1991; Schmitz \& Fleck 1995; Sutmann \& Ulmschneider 1995a,b), where the word "resonance" was used in a sense of "echo". Since in typical resonance phenomena one would expect the wave velocity $v(z, t)$ to become unbounded when $\omega$ approaches $\Omega_{\mathrm{T}}$, and since the latter never happens (see Eq. (12)), we follow Kalkofen et al. (1994) and call these oscillations free atmospheric oscillations throughout this paper.

\subsection{Excitation by a spectrum of longitudinal tube waves}

We assume that a spectrum of these waves is generated at the lower tube boundary $(z=0)$ and propagates upward along the tube. The spectrum is approximated here by a linear superposition of sinusoidal partial waves with different amplitudes, frequencies and phases. This allows writing the lower boundary condition $v_{\mathrm{o}}(t)$ as

$v_{\mathrm{o}}(t)=\sum_{n=1}^{N} u_{n} \mathrm{e}^{-\mathrm{i}\left(\omega_{n} t+\varphi_{n}\right)}$,

where $u_{n}$ and $\omega_{n}$ are the velocity amplitudes and frequencies of the partial waves, respectively, and $\varphi_{n}$ are arbitrary constant phases. With this boundary condition, we obtain (see Eq. (36) in Sutmann et al. 1998)

$$
\begin{aligned}
v(t, z)= & \sum_{n=1}^{N} u_{n} \mathrm{e}^{-\mathrm{i} \varphi_{n}} \mathrm{e}^{-\mathrm{i}\left(\omega_{n} t-\sqrt{\omega_{n}^{2}-\Omega_{\mathrm{T}}^{2}} z / c_{\mathrm{T}}\right)} \\
& +\sum_{n=1}^{N} u_{n} \mathrm{e}^{-\mathrm{i} \varphi_{n}} \sqrt{\frac{2 \Omega_{\mathrm{T}}}{\pi}} \frac{1}{\omega_{n}^{2}-\Omega_{\mathrm{T}}^{2}} \frac{z}{c_{\mathrm{T}}} \frac{1}{t^{3 / 2}} \\
& \times\left[\Omega_{\mathrm{T}} \sin \left(\Omega_{\mathrm{T}} t-\frac{3 \pi}{4}\right)+\mathrm{i} \omega_{n} \cos \left(\Omega_{\mathrm{T}} t-\frac{3 \pi}{4}\right)\right] .
\end{aligned}
$$

There are obvious similarities to the previous case of monochromatic waves. The first sum on the RHS of the above equation describes the forced atmospheric oscillations that are periodic and non-decaying in time. All partial waves with $\omega_{n}>\Omega_{\mathrm{T}}$ are propagating waves, however, partial waves with $\omega_{n}<\Omega_{\mathrm{T}}$ are evanescent waves and their amplitudes decrease exponentially with height. The free atmospheric oscillations with the cutoff frequency $\Omega_{\mathrm{T}}$ are described by the second term on the RHS of Eq. (14). It is seen that each partial wave excites its own free oscillations, which superpose and have amplitudes that decay in time as $t^{-3 / 2}$. If $\omega_{n} \rightarrow \Omega_{\mathrm{T}}$, then we must take $t \rightarrow \infty$, which makes the second term in Eq. (14) equal zero and the free and forced oscillations become the same and they do not decay in time.

\subsection{Excitation by a $\delta$-function pulse}

We now assume that one longitudinal pulse with a $\delta$-function shape is being generated by the external turbulence at $z=0$. Here, the required boundary condition is

$v_{\mathrm{o}}\left(t^{\prime}\right)=u_{\mathrm{o}} \delta\left(t^{\prime}\right)$,

where $t^{\prime}=\Omega_{\mathrm{T}} t / 2 \pi$.
Using Eq. (9) and applying the limit of $t \gg z / c_{\mathrm{T}}$ to Eq. (10), the solution can be written (see Eq. (41) in Sutmann et al. 1998) as

$v(t, z)=-u_{\mathrm{o}} \sqrt{\frac{2 \pi}{\Omega_{\mathrm{T}}}} \frac{z}{c_{\mathrm{T}}} \frac{1}{t^{3 / 2}} \cos \left(\Omega_{\mathrm{T}} t-\frac{3 \pi}{4}\right)$,

which shows that the $\delta$-function pulse excites only the free atmospheric oscillations that decay in time in the same manner $\left(t^{-3 / 2}\right)$ as the free oscillations generated by longitudinal tube waves (see Eq. (12)). The main reason that the forced oscillations are not present is that there is no continuous excitation.

\subsection{Excitation by a wavetrain of random pulses}

Finally, we consider the response of the flux tube to a wavetrain of longitudinal pulses with randomly chosen amplitudes and periods. The wavetrain is introduced at the lower boundary of the tube $(z=0)$ where after each completed wave period a new period and a new amplitude are stochastically chosen. This boundary condition requires $v_{\mathrm{o}}(t)$ to be given in the following form:

$v_{\mathrm{o}}(t)=\sum_{n=1}^{\infty} u_{n} \mathrm{e}^{-\mathrm{i} \omega_{n} t}\left[\mathcal{H}\left(t-t_{n-1}\right)-\mathcal{H}\left(t-t_{n}\right)\right]$,

where $u_{n}$ and $\omega_{n}$ are randomly chosen wave amplitudes and periods, respectively. In addition, we have

$t_{n}=\sum_{i=0}^{n} T_{i}, \quad t_{n-1}=\sum_{i=0}^{n-1} T_{i}$,

and

$T_{0}=0, \quad T_{i}=\frac{2 \pi}{\omega_{i}}$.

Using Eq. (9) and applying the asymptotic limit of $t \gg z / c_{\mathrm{T}}$ to Eq. (10), we obtain (see Eq. (62) in Sutmann et al. 1998)

$$
\begin{aligned}
v(t, z)= & \sqrt{\frac{2 \Omega_{\mathrm{T}}}{\pi}} \frac{z}{c_{\mathrm{T}}} \sum_{n=1}^{N} u_{n} \frac{1}{\omega_{n}^{2}-\Omega_{\mathrm{T}}^{2}} \frac{2}{t^{3 / 2}} \\
& \times\left[\Omega_{\mathrm{T}} \cos \left(\Omega_{\mathrm{T}} t-\varphi_{1}\right)-\mathrm{i} \omega_{n} \sin \left(\Omega_{\mathrm{T}} t-\varphi_{1}\right)\right] \sin \varphi_{2},
\end{aligned}
$$

where $N$ is chosen in such a way that $t>t_{N}$, and

$\varphi_{1}=\frac{1}{2} \Omega_{\mathrm{T}}\left(t_{n}+t_{n-1}\right)+\frac{3 \pi}{4}$

$\varphi_{2}=\frac{1}{2} \Omega_{\mathrm{T}} \Delta t_{n}$,

with $\Delta t_{n}=t_{n}-t_{n-1}$.

The obtained result is similar to that found for the excitation by a $\delta$-function pulse (see Eq. (16)) because it shows that only the free atmospheric oscillations exist. Obviously, this is an expected result because the excitation is not continuous but instead it is stopped at times $t>t_{N}$, which means that only $N$ number of pulses are generated. As a result of this wavetrain with finite number of pulses both the forced and free oscillations are excited, however, for the analytical solution to be valid we need $t \gg t_{n-1}, t \gg t_{n}$ and $t \gg z / c_{\mathrm{T}}$, so that 
Table 1. The characteristic velocity $c_{\mathrm{T}}\left(\mathrm{km} \mathrm{s}^{-1}\right)$, cutoff frequency $\Omega_{\mathrm{T}}\left(\mathrm{s}^{-1}\right)$ and total wave energy flux $F_{\mathrm{T}}\left(\mathrm{erg} \mathrm{cm}^{-2} \mathrm{~s}^{-1}\right)$ are given for three different values of $B_{\mathrm{o}} / B_{\mathrm{eq}}$ used in this paper. By comparing the maximum wave frequency $\omega_{\max }$ to $\Omega_{\mathrm{T}}$, it is seen that most of the generated wave energy is carried by propagating longitudinal tube waves. The fluxes $F_{\mathrm{T}}$ are computed following Musielak et al. (1995) but using 32 frequency points (Musielak \& Ulmschneider 2001).

\begin{tabular}{ccccc}
\hline \hline$B_{\mathrm{o}} / B_{\mathrm{eq}}$ & $c_{\mathrm{T}}$ & $\Omega_{\mathrm{T}}$ & $\omega_{\max }$ & $F_{\mathrm{T}}$ \\
\hline 0.75 & 5.7 & 0.0315 & 0.0697 & $6.5 \times 10^{7}$ \\
0.85 & 6.4 & 0.0317 & 0.0784 & $2.8 \times 10^{7}$ \\
0.95 & 7.0 & 0.0319 & 0.0862 & $7.0 \times 10^{6}$ \\
\hline
\end{tabular}

the forced atmospheric oscillations are no longer present inside the tube. Hence, the analytical solution describes only the free atmospheric oscillations and these oscillations decay in time as $t^{-3 / 2}$, which is the same dependence as found for all other excitation types discussed in this paper.

\section{Application to solar magnetic flux tubes}

To compute the structure of the solar atmosphere outside the magnetic flux tube, we take the gravity $g=2.376 \times 10^{4} \mathrm{~cm} / \mathrm{s}^{2}$ and the gas pressure $p_{\mathrm{e}}=4 \times 10^{4} \mathrm{dyn} / \mathrm{cm}^{2}$, which corresponds approximately to the temperature $T_{\mathrm{e}}=5000 \mathrm{~K}$ in the VAL model (Vernazza et al. 1981); the atmospheric height corresponding to this pressure and temperature is identified as $z=0$ in our model. The atmosphere outside the tube is assumed to be isothermal with $T_{\mathrm{e}}=5000 \mathrm{~K}$ and in hydrostatic equilibrium. The atmosphere inside the tube is also taken to be isothermal with $T_{\mathrm{o}}=T_{\mathrm{e}}$ and extends from $z=0$ to $z=2000 \mathrm{~km}$. We specify the tube magnetic field strength by $B_{\mathrm{o}} / B_{\text {eq }}=0.85$ at $\tau_{5000}=1$ (see Sect. 3 ) and use the horizontal pressure balance to calculate the gas pressure and density inside the tube at the height $z=0 \mathrm{~km}$. Magnetic flux conservation and the horizontal pressure balance allow then to compute the tube's exponentially spreading field geometry and physical parameters over the entire height range. Since the strength of the tube field varies for flux tubes on the Sun (e.g., Solanki 1993), we also consider $B_{\mathrm{o}} / B_{\text {eq }}=0.75$ and 0.95 (see e.g., Ulmschneider $\&$ Musielak 1998).

For each value of the tube magnetic field strength, we compute the characteristic tube speed $c_{\mathrm{T}}$ and the cutoff frequency $\Omega_{\mathrm{T}}$ (see Table 1). Taking $\gamma=5 / 3$, we find $c_{\mathrm{S}}=7.3 \mathrm{~km} \mathrm{~s}^{-1}$ and $\Omega_{\mathrm{S}}=0.0312 \mathrm{~s}^{-1}$ or $\nu_{\mathrm{S}}=\Omega_{\mathrm{S}} / 2 \pi=4.9 \mathrm{mHz}$. Comparison of the values of $c_{\mathrm{S}}$ and $c_{\mathrm{T}}$ shows that longitudinal tube waves propagate with a speed that is lower than the sound speed in our tube models. This is consistent with Eq. (5) as $B_{\mathrm{o}} / B_{\text {eq }}=0.75$, 0.85 and 0.95 correspond to $\beta \approx 0.8,0.4$ and 0.1 , respectively. In addition, we find that the values of $\Omega_{\mathrm{T}}$ for the three considered tube magnetic fields are very similar to the acoustic cutoff frequency $\Omega_{\mathrm{S}}$ (see Eq. (6)), which means that the free atmospheric oscillations inside and outside the tube will have almost the same frequencies. Note also that in our approach, $c_{\mathrm{T}}, c_{\mathrm{S}}$, $\Omega_{\mathrm{T}}$ and $\Omega_{\mathrm{S}}$ are constant, because the background atmosphere is isothermal and because both the gas density and the tube magnetic field decrease with height, so that $c_{\mathrm{A}}=B_{\mathrm{o}} / \sqrt{4 \pi \rho_{\mathrm{o}}}$ remains constant.
In the following, we present the results for the free atmospheric oscillations by plotting the variation of the real part of the normalized wave velocity $v(z, t) / c_{\mathrm{T}}$ with time at two different atmospheric heights, $z=500$ and $2000 \mathrm{~km}$. In our calculations the conditions $t \gg z / c_{\mathrm{T}}$ and $t \gg 1 /\left|\Omega_{\mathrm{T}} \pm \omega\right|$ are always fulfilled.

\subsection{Monochromatic waves}

It remains to specify the amplitude of our wave disturbances which are thought to arise from the stochastic motions which buffet the flux tube in the convection zone. An analytical method describing the interaction between a thin and vertically oriented magnetic flux tube and the external turbulent motions has been developed by Musielak et al. (1989, 1995) who considered the generation of longitudinal tube waves by these motions in the solar and stellar convection zones. The method is based on the original work by Lighthill (1952) in which the inhomogeneous wave equation is derived and the source function is assumed to be fully determined by the turbulent motions; the latter are prescribed by using an extended form of the Kolgomorov turbulent energy spectrum and a modified Gaussian frequency factor (Musielak et al. 1994). To compute the wave energy spectra and fluxes resulting from this theory, one must specify the following solar parameters: gravity (see above), the effective temperature $\left(T_{\text {eff }}=5770 \mathrm{~K}\right)$ and metallicity $\left(Z_{\mathrm{m}}=0.02\right)$. In addition, we must know the mixinglength parameter $\alpha$, which is assumed to be 2 in all our calculations, and the strength of the tube magnetic field that we take $B_{\mathrm{o}} / B_{\text {eq }}=0.75,0.85$ and 0.95 .

The newly computed wave energy spectra for the Sun are presented in Fig. 1, which shows that the amount of generated wave energy decreases with increasing magnetic field strength (see also Musielak et al. 1989). The total fluxes $F_{\mathrm{T}}$ carried by longitudinal tube waves propagating inside solar magnetic flux tubes are calculated by intergrating the computed spectra over frequency (see Table 1); note that $\omega_{\max }$ corresponds to the maximum flux of these spectra and is much higher than the cutoff $\Omega_{\mathrm{T}}$, which means that the generated waves are always propagating waves.

We begin with the excitation of the free atmospheric oscillations by monochromatic longitudinal tube waves described by Eq. (11). The frequency of these waves is assumed to be $\omega=\omega_{\max }$ (see Table 1) and the initial amplitude is given by $u_{\mathrm{o}}=\sqrt{2 F_{\mathrm{T}} / \rho_{\mathrm{o}} c_{\mathrm{T}}}$, where the total wave energy flux $F_{\mathrm{T}}$ is taken from Table 1 . The fact that the total wave energy flux is carried by a monochromatic wave is a rather crude approximation but we compute this case here because we want to compare it with the more realistic case when the entire wave energy spectrum is considered (see below). Taking the real part of Eq. (12) and normalizing $v(t, z)$ by $c_{\mathrm{T}}$, we compute the time evolution of the free atmospheric oscillations at the atmospheric heights $z=500$ and $z=2000 \mathrm{~km}$. The results are shown in Fig. 2. It is seen that the amplitude of these oscillations is small compared to the tube speed $c_{\mathrm{T}}$ and quickly decreases with time. The amplitude slightly increases when the magnetic field strength decreases, because the total wave energy flux increases (see Table 1), 


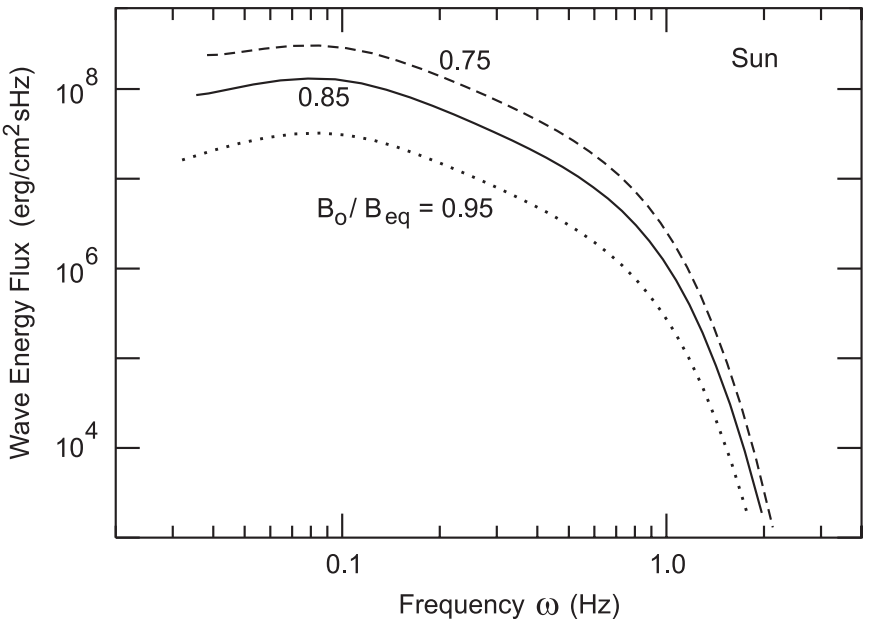

Fig. 1. Longitudinal wave energy fluxes as function of circular frequency $\omega$ for three different values of the tube magnetic field strength: $B_{\mathrm{o}} / B_{\mathrm{eq}}=0.75,0.85$ and 0.95 specified at $\tau_{5000}=1$.

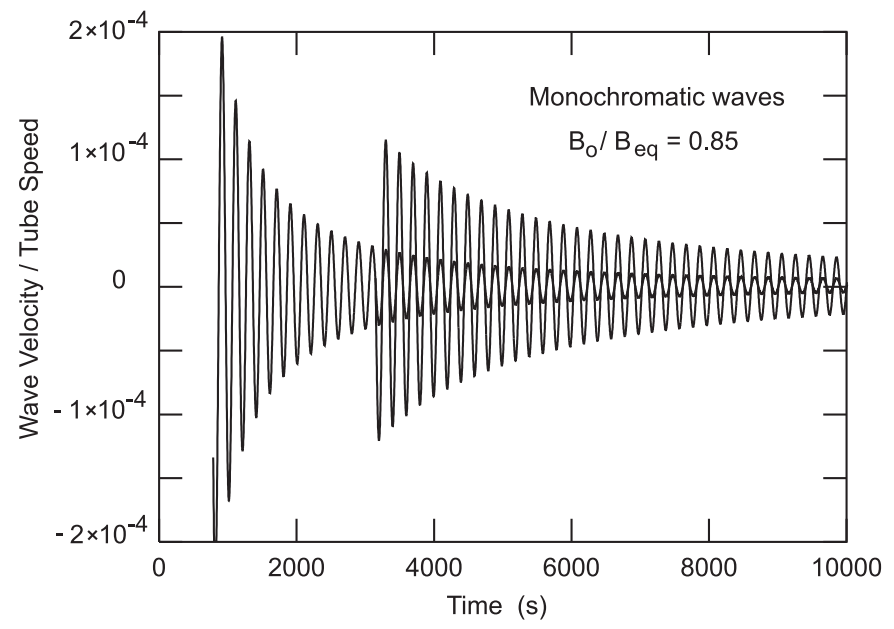

Fig. 2. Time evolution of the free atmospheric oscillations inside solar magnetic flux tubes with $B_{\mathrm{o}} / B_{\mathrm{eq}}=0.85$. The oscillations are driven by monochromatic longitudinal tube waves with $\omega=0.0784$ and $F_{\mathrm{T}}=$ $2.8 \times 10^{7}\left(\mathrm{erg} / \mathrm{cm}^{2} \mathrm{~s}\right)$, and the real part of $v(t, z) / c_{\mathrm{T}}$ (see Eq. (12)) is plotted at two different atmospheric heights $z=500 \mathrm{~km}$ and $z=$ $2000 \mathrm{~km}$.

but this effect is never significant. Since the oscillations shown in Fig. 2 are calculated for $B_{\mathrm{o}} / B_{\text {eq }}=0.85$, the period of the oscillations is approximately $3 \mathrm{~min}\left(=2 \pi / \Omega_{\mathrm{T}}\right)$. As the changes in the values $\Omega_{\mathrm{T}}$ given in Table 1 are very small, this means that the frequency of the free atmospheric oscillations is practically the same for the entire considered range of tube magnetic field strengths.

\subsection{A spectrum of waves}

We now discuss the excitation of free atmospheric oscillations by a spectrum of longitudinal tube waves (see Eq. (13)) propagating inside the magnetic flux tubes. As discussed in Sect. 3.2, this spectrum consists of a series of partial waves. To specify the amplitude of these waves, we use the full wave energy spectra generated in the solar convection zone

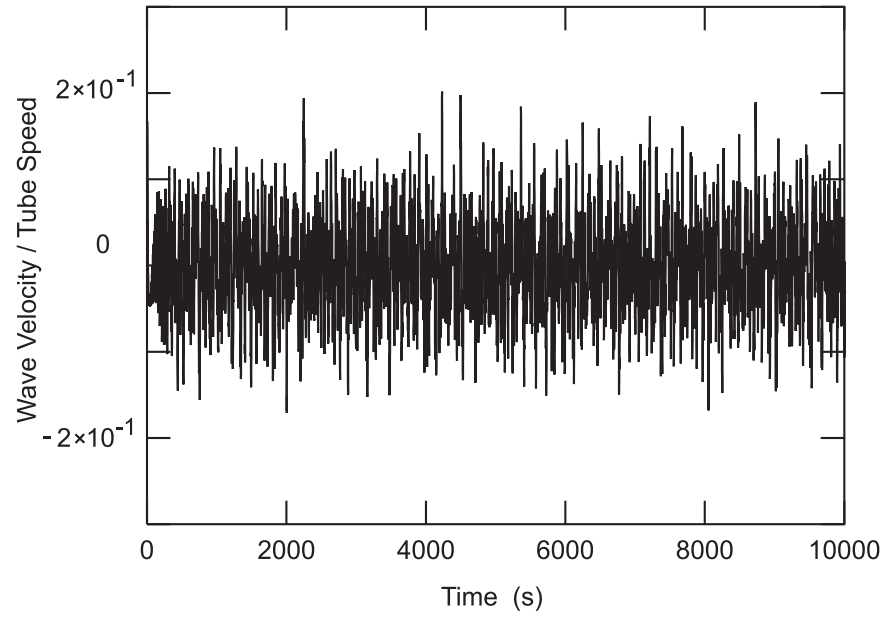

Fig. 3. Time evolution of the initial wave velocity $v_{0}(t)$ normalized by $c_{\mathrm{T}}$. These velocity fluctuations represent the spectrum of longitudinal tube waves shown in Fig. 1 (solid line) at the atmospheric height $z=0 \mathrm{~km}$ inside solar magnetic flux tubes with $B_{\mathrm{o}} / B_{\mathrm{eq}}=0.85$.

(see Fig. 1). During their propagation along the magnetic flux tubes, these waves excite free atmospheric oscillations with the cutoff frequency $\Omega_{\mathrm{T}}$ but they also dissipate their energy and heat the background atmosphere. Since our wave energy spectra are computed for 32 frequency points (following Musielak $\&$ Ulmschneider 2001), we identify these frequencies with $\omega_{n}$ of Eq. (13). For each frequency, we calculate the intial wave amplitude $u_{n}=\sqrt{2 F_{n} / \rho_{\mathrm{o}} c_{\mathrm{T}}}$, where $F_{n}$ is the wave energy flux integrated over the frequency interval $\Delta \omega=\omega_{n+1}-\omega_{n}$. Because these waves are propagating $\left(\omega_{n}>\Omega_{\mathrm{T}}\right)$, we assume that all of them have the same phase $\phi_{\mathrm{n}}=0$. A typical time evolution of the initial wave velocity $v_{\mathrm{o}}(t, z=0)$ given by Eq. (13) for a wave spectrum with $B_{\mathrm{o}} / B_{\mathrm{eq}}=0.85$ is shown in Fig. 3; it is seen that some spikes have amplitudes as large as $0.2 c_{\mathrm{T}}$. In similar plots for $B_{\mathrm{o}} / B_{\text {eq }}=0.75$ and 95 , one finds that the spikes become higher (up to $0.3 c_{\mathrm{T}}$ ) and lower $\left(0.15 c_{\mathrm{T}}\right)$, respectively, which reflects the amount of wave energy generated in these two cases (see Fig. 1 and Table 1).

We take the real part of Eq. (14) and calculate the time evolution of the free atmospheric oscillations for $B_{\mathrm{o}} / B_{\mathrm{eq}}=0.75$, 0.85 and 0.95 . The results obtained for the first two field strengths are presented in Figs. 4 and 5. It is seen that the oscillations driven by wave spectra also decay in time as $t^{-3 / 2}$, however, that their amplitudes are much higher than those for monochromatic waves (see Fig. 2). This is explained by the fact that some partial waves of the spectrum have amplitudes higher (see Fig. 2) than the amplitude of monochromatic waves; the latter is $u_{\mathrm{o}}=0.06 c_{\mathrm{T}}$ for $B_{\mathrm{o}} / B_{\text {eq }}=0.85$. By comparing Figs. 4 and 5, one finds that the amplitude of the oscillations increases with decreasing magnetic field strength, but that these changes are small. The frequency of the free atmospheric oscillations inside solar magnetic flux tubes is found to be approximately $3 \mathrm{~min}$ and is relatively insensitive to the magnetic field strength (see Table 1). It must also be noted that the spectrum of waves is continuously generated in the solar convection zone, which means that the oscillations should be 


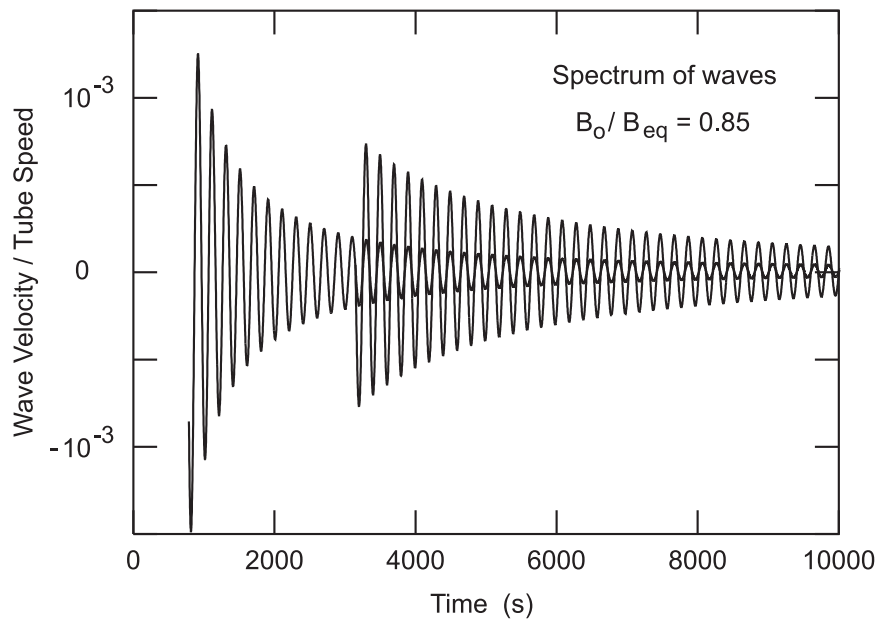

Fig. 4. Time evolution of free atmospheric oscillations inside solar magnetic flux tubes with $B_{\mathrm{o}} / B_{\mathrm{eq}}=0.85$. The oscillations are driven by the spectrum of longitudinal tube waves shown in Fig. 1 and only the real part of $v(t, z) / c_{\mathrm{T}}$ is plotted at two different atmospheric heights $z=500 \mathrm{~km}$ and $z=2000 \mathrm{~km}$.

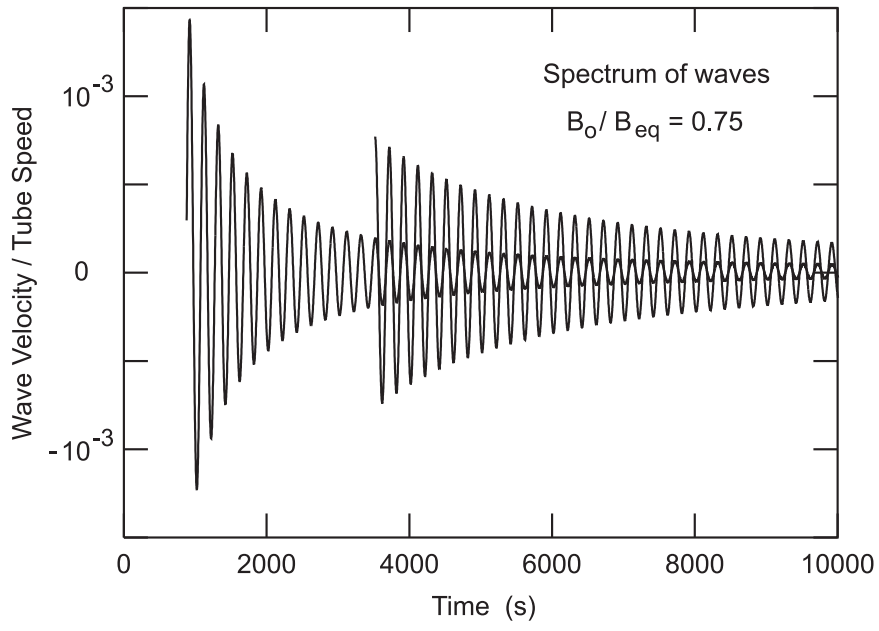

Fig. 5. The same as Fig. 4 but for $B_{\mathrm{o}} / B_{\text {eq }}=0.75$.

always present inside the tubes despite their decay in time (e.g., Sutmann \& Ulmschneider 1995b).

\subsection{A wavetrain of random pulses}

Finally, we consider the wavetrain with a finite number of $N$ sinusoidal pulses with randomly chosen amplitudes and frequencies at times $t>t_{N}$. In this case a pulse with a new period and new amplitude is introduced whenever the previous pulse has been completed. Frequencies of these pulses are always a fraction of $\Omega_{\mathrm{T}}$ but no pulses with $\omega_{n}=\Omega_{\mathrm{T}}$ are allowed. Similarly, their amplitudes are also limited and not allowed to be higher than $0.1 c_{\mathrm{T}}$. The source of these pulses can be rapid horizontal motions which occur at the top of the solar convection zone and in the solar photosphere (e.g., Muller et al. 1994; Solanki et al. 1996; Steiner et al. 1998). Without getting into details how these pulses are actually excited (e.g., Ulmschneider $\&$ Musielak 1998), we simply assume here that we have at $z=0$ the random pulses described by Eq. (17), and that they

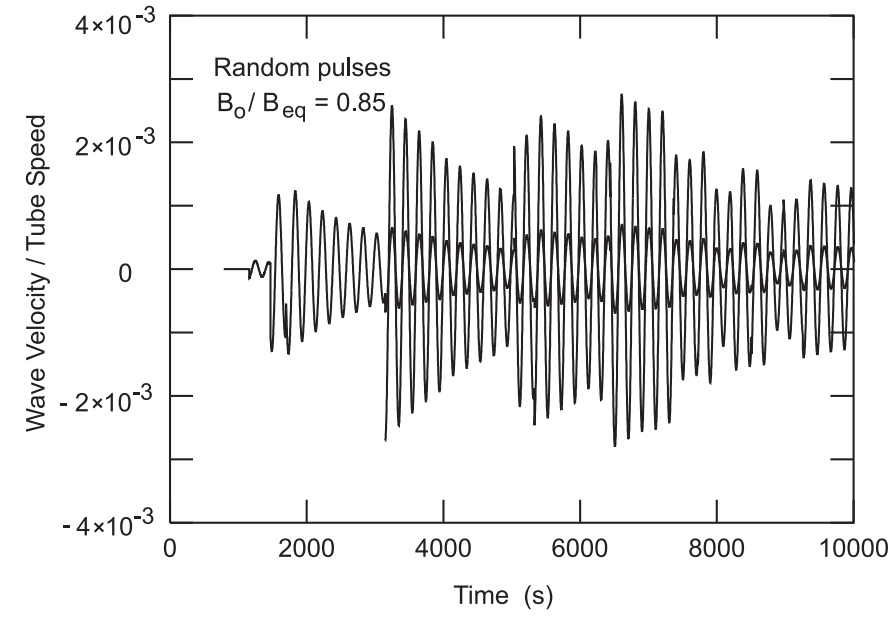

Fig. 6. Time evolution of the free atmospheric oscillations inside solar magnetic flux tubes with $B_{\mathrm{o}} / B_{\mathrm{eq}}=0.85$. The oscillations are driven by a wavetrain of longitudinal pulses with randomly chosen amplitudes and frequencies. The real part of $v(t, z) / c_{\mathrm{T}}$ is plotted at two different atmospheric heights $z=500 \mathrm{~km}$ and $z=2000 \mathrm{~km}$.

propagate upward producing the free atmospheric oscillations inside the tube. Since the wavetrain is finite only free oscillations are present at $t>t_{N}$ (see Eq. (20)). The time evolution is plotted in Fig. 6. Clearly, each random pulse decays in time as $t^{-3 / 2}$, even so the oscillations are sustained in the atmosphere for at least $10000 \mathrm{~s}$, which is the time of our calculations. If these random pulses were continuously generated, then the free atmospheric oscillations would be permanently present inside solar magnetic flux tubes. Note also that the amplitudes of these oscillations are comparable to those shown in Fig. 4 for the excitation by the spectrum of waves.

\section{Discussion and conclusions}

The presented results clearly imply that free atmospheric oscillations should always be present inside solar magnetic flux tubes. The period of these oscillations is approximately $3 \mathrm{~min}$ and is practically independent of the strength of the magnetic field in the tube. Because of physical similarities between longitudinal tube waves and acoustic waves, their cutoff periods are also very similar and $P_{\mathrm{T}} \approx P_{\mathrm{S}} \approx 3 \mathrm{~min}$, which means that the free atmospheric oscillations inside and outside the tubes are almost identical.

The oscillations inside the tubes can be driven either by a spectrum of longitudinal tube waves continuously generated in the solar convection zone or by wavetrains of random pulses which continuously propagate inside these tubes. The amplitude of these oscillations is relatively low $v(t, z) \approx 10^{-3} c_{\mathrm{T}}$ and quickly decays with time at each atmospheric height. Despite these facts, the oscillations should be observable, because they are expected to be constantly reexcited.

Our investigation is for exponentially spreading tubes in appropriate regions of the chromospheric network at the boundary of superganulation cells where enough space is available before the tubes meet neighboring flux tubes. 
The results are also valid for intracell flux tubes, that is tubes in the interior of the supergranulation cells. The observed 3 min oscillations in the interior of these cells can be explained both by processes in magnetic flux tubes and outside such structures. Moreover, it is unsafe to conclude from the absence of chromospheric bright point emission in the cell interior, that magnetic flux tubes are not present, because these exponentially spreading tubes are very insufficiently heated (Fawzy et al. 1998). These latter calculations, however, do not take into account the influx of acoustic energy from outside the tube.

Our calculations using longitudinal tube waves and pulses do not agree with the observed oscillations in the range from 6 to 15 min (e.g., Kalkofen 1996). Therefore, the 7-min oscillations must be of different nature and origin than the oscillations discussed in this paper (see Kalkofen 1997; Hasan \& Kalkofen 1999; also Paper II of this series).

Our conclusions about the nature of the generated oscillations are summarized as follows:

1. The excitation of an isothermal atmosphere inside solar magnetic flux tubes by longitudinal tube waves and pulses leads to the free atmospheric oscillations with periods near $3 \mathrm{~min}$. The frequency of these oscillations is the cutoff frequency $\Omega_{\mathrm{T}}$ for these waves which is very similar to, and essentially indistinguishable from the acoustic cutoff frequency $\Omega_{\mathrm{S}}$. In addition, $\Omega_{\mathrm{T}}$ is practically independent of the field strength in the magnetic tube.

2. The free atmospheric oscillations decay in time as $t^{-3 / 2}$ if the frequency of driving waves is not equal to the cutoff $\Omega_{\mathrm{T}}$. This time dependence is the same for all considered mechanisms of the excitation of these oscillations.

3. For the continuous excitation, the forced atmospheric oscillations are also present in the atmosphere. Different from the free oscillation they do not decay in time and their frequency is the same as the frequency of the driving waves. However, if the latter is equal to the cutoff $\Omega_{\mathrm{T}}$, then the forced and free oscillations are identical and they do not decay in time. When the atmosphere is stochastically and perpetually excited by longitudinal wave pulses, the generated free atmospheric oscillations with frequency $\Omega_{\mathrm{T}} \approx \Omega_{\mathrm{S}}$ will always be present.

4. The amplitude of the free atmospheric oscillations is a small fraction of the tube speed $c_{\mathrm{T}}$ and it is larger for the excitation by a spectrum of waves and random pulses than for monochromatic waves. This amplitude increases when the tube magnetic field is decreased.

5. The observed 3-min oscillations inside the supergranulation cells are consistent both with nonmagnetic free atmospheric oscillations and with free oscillations in magnetic flux tubes. Longitudinal waves and pulses do not explain the observed 7-min oscillations in the chromospheric network.

Acknowledgements. This work was supported by NSF under grant ATM-0087184 (Z.E.M. and P.U.), by the DFG grant U157/25-3, and by NATO under grant CRG-910058 (P.U. and Z.E.M.). Z.E.M. also acknowledges the support of this work by the Alexander von Humboldt Foundation.

\section{References}

Bogdan, T. J. 1992, NATO ASI Ser. C, ed. J. H. Thomas, \& N. O. Weiss (Dordrecht: Kluwer), 375, 345

Bogdan, T. J. 1994, in Solar Magnetic Fields, ed. M. Schüssler, \& W. Schmidt (Cambridge: Cambridge Univ. Press), 229

Davies, B. 1978, Integral Transforms and Their Applications (New York: Springer-Verlag)

Curdt, W., \& Heinzel, P. 1998, ApJ, 503, L95

Damé, L. 1983, Thèse, Université de Paris VII

Defouw, R. J. 1976, ApJ, 209, 266

Deubner, F. L. 1991, in Mechanisms of Chromospheric and Coronal Heating, ed. P. Ulmschneider, E. R. Priest, \& R. Rosner (Berlin: Springer), 6

Deubner, F. L., \& Fleck, B. 1990, A\&A, 228, 506

Edwards, C. H., \& Penney, D. E. 1989, Elementary Differential Equations with Boundary Value Problems (New Jersey: Prentice-Hall, Englewood Cliffs), 137

Fawzy, D., Ulmschneider, P., \& Cuntz, M. 1998, A\&A, 336, 1029

Fleck, B., \& Schmitz, F. 1991, A\&A, 250, 235

Fleck, B., \& Schmitz, F. 1993, A\&A, 273, 671

Haberman, R. 1987, Elementary Applied Partial Differential Equations (New Jersey: Prentice-Hall, Englewood Cliffs), 246

Hasan, S. S., \& Kalkofen, W. 1999, ApJ, 519, 899

Kalkofen, W. 1996, ApJ, 468, L69

Kalkofen, W. 1997, ApJ, 486, L145

Kalkofen, W., Rossi, P., Bodo, G., \& Massaglia, S. 1994, A\&A, 284, 976

Lamb, H. 1908, Proc. Lond. Math. Soc., Ser., 2, 7, 122

Leibacher, J. W., \& Stein, R. F. 1971, ApJ, 7, L191

Lighthill, M. J. 1952, Proc. Roy. Soc. London, A211, 564

Lites, B. W., Rutten, R. J., \& Kalkofen, W. 1993, ApJ, 414, 345

Muller, R., Roudier, Th., Vigneau, J., \& Auffret, H. 1994, A\&A, 283, 232

Musielak, Z. E., Rosner, R., \& Ulmschneider, P. 1989, ApJ, 337, 470

Musielak, Z. E., Rosner, R., Stein, R. F., \& Ulmschneider, P. 1994, ApJ, 423, 474

Musielak, Z. E., Rosner, R., Gail, H. P., \& Ulmschneider, P. 1995, ApJ, 448,865

Musielak, Z. E., Rosner, R., \& Ulmschneider, P. 2000, ApJ, 541, 410

Musielak, Z. E., Rosner, R., \& Ulmschneider, P. 2002, ApJ, 573, 418

Musielak, Z. E., \& Ulmschneider, P. 2001, A\&A, 370, 541

Musielak, Z. E., \& Ulmschneider, P. 2003, submitted (Paper II)

Rae, I. C., \& Roberts, B. 1982, ApJ, 256, 761

Rutten, R. J., \& Uitenbroek, H. 1991, Sol. Phys., 134, 15

Schmitz, F., \& Fleck, B. 1995, A\&A, 301, 483

Solanki, S. K. 1993, Space Sci. Rev., 63, 1

Solanki, S. K., Rüedi, I., Bianda, M., \& Steffen, M. 1996, A\&A, 308, 623

Spruit, H. C., \& Roberts, B. 1983, Nature, 304, 401

Steiner, O., Grossmann-Doerth, Knölker, M., \& Schüssler, M. 1998, ApJ, 495, 468

Stenflo, J. O. 1994, Solar Magnetic Fields (Dordrecht: Kluwer)

Sutmann, G., Musielak, Z. E., \& Ulmschneider, P. 1998, A\&A, 340, 556

Sutmann, G., \& Ulmschneider, P. 1995a, A\&A, 294, 232

Sutmann, G., \& Ulmschneider, P. 1995b, A\&A, 294, 241

Ulmschneider, P., Fawzy, D. E., Musielak, Z. E., \& Stępień, K. 2001, ApJ, 559, L167

Ulmschneider, P., \& Musielak, Z. E. 1998, A\&A, 338, 311

Ulrich, R. K. 1970, ApJ, 162, 993

Vernazza, J. E., Avrett, E. H., \& Loeser, R. 1981, ApJS, 45, 635

Wang, J., Wang, H., Tang, F., Lee, J. W., \& Zirin, H. 1995, Sol. Phys., 160,277 